\title{
Clinicopathologic Correlations of E-cadherin and Prrx-1 Expression Loss in Hepatocellular Carcinoma
}

Kijong Yi · Hyunsung Kim Yumin Chung · Hyein Ahn Jongmin Sim · Young Chan Wi Ju Yeon Pyo · Young-Soo Song Seung Sam Paik · Young-Ha Oh

Department of Pathology, Hanyang University College of Medicine, Seoul, Korea

Received: January 25, 2016

Revised: June 16, 2016

Accepted: June 21, 2016

Corresponding Author

Young-Ha Oh, MD

Department of Pathology, Hanyang University

College of Medicine, 222-1 Wangsimni-ro,

Seongdong-gu, Seoul 04763, Korea

Tel: +82-31-560-2498

Fax: +82-31-560-2339

E-mail: yhoh@hanyang.ac.kr

\begin{abstract}
Background: Developing predictive markers for hepatocellular carcinoma (HCC) is important, because many patients experience recurrence and metastasis. Epithelial to mesenchymal transition (EMT) is a developmental process that plays an important role during embryogenesis and also during cancer metastasis. Paired-related homeobox protein 1 (Prrx-1) is an EMT inducer that has recently been introduced, and its prognostic significance in HCC is largely unknown. Methods: Tissue microarray was constructed using surgically resected primary HCCs from 244 cases. Immunohistochemical staining of E-cadherin and Prrx-1 was performed. The correlation between Ecadherin loss and Prrx-1 expression, as well as other clinicopathologic factors, was evaluated. Results: E-cadherin expression was decreased in 96 cases (39.4\%). Loss of E-cadherin correlated with a higher recurrence rate $(p<.001)$ but was not correlated with patient's survival. Thirtytwo cases (13.3\%) showed at least focal nuclear Prrx-1 immunoreactivity while all non-neoplastic livers $(n=22)$ were negative. Prrx-1 expression was not associated with E-cadherin loss, survival or recurrence rates, pathologic factors, or the Ki-67 labeling index. Twenty tumors that were positive for E-cadherin and Prrx-1 had significantly higher nuclear grades than the rest of the cohort $(p$ $=.037$ ). In Cox proportional hazard models, E-cadherin loss and large vessel invasion were independent prognostic factors for shorter disease-free survival. Cirrhosis and high Ki-67 index (> $40 \%$ ) were independent prognostic factors for shorter overall survival. Conclusions: Prrx-1 was expressed in small portions of HCCs but not in normal livers. Additional studies with a large number of Prrx-1-positive cases are required to confirm the results of this study.
\end{abstract}

Key Words: Liver; Neoplasms; Epithelial-mesenchymal transition; Prrx-1 protein
Hepatocellular carcinoma (HCC) is one of the most lethal and relatively prevalent cancers worldwide, as well as in Korea. The Korean National Cancer Information Center estimates that over 2.2 per hundred thousand persons were killed by HCC in 2013, which is the second most common cause of death by cancer in Korea. ${ }^{1}$ Although surgical resection is effective in cases of the incipient disease, even in early stages, a considerable number of patients experience recurrence and metastasis leading to a poor prognosis. ${ }^{2}$ Therefore, it is important to develop markers that indicate poor prognosis, and to provide stratified follow-up guidelines for clinicians.

Epithelial tumor metastasis can be described by a developmental program called epithelial to mesenchymal transition (EMT), which plays crucial roles in embryonic development, including mesoderm formation and migration of neural crest cells. ${ }^{3}$ During metastasis, the tumor cells lose their epithelial property, gain migratory activity, disseminate through the blood stream, and finally, establish metastatic foci in distant organs. ${ }^{3}$
Paired-related homeobox protein 1 (Prrx-1; PRX-1) is a transcription factor that has recently been found to induce EMT in embryogenesis, as well as in several cancers, including breast, pancreas, colon, and thyroid cancer. ${ }^{4-7}$ However, it has been inconsistently reported on whether its upregulation or downregulation is associated with poor prognosis. In colorectal and thyroid cancers, overexpression of Prrx-1 was associated with poor prognosis or worse histologic type. ${ }^{4,7}$ In comparison, in breast cancers and HCCs, downregulation of Prrx-1 was associated with poor prognosis. ${ }^{5,8}$

In this study, we used tissue microarray (TMA) to determine if the loss of E-cadherin is a prognostic marker for predicting early recurrence and poor survival rate. We surgically obtained 244 cases of human HCC tissues in the Hanyang University Medical Center, Seoul and Guri hospitals. We examined Prrx-1 expression in HCC using immunohistochemistry, and investigated the relationship between its expression and loss of E-cadherin. We also worked to determine if expression of Prrx-1 could 
be a prognostic marker to predict metastasis or poor survival rate, because it induces EMT and its significance is currently unknown. We also investigated the relationship between clinicopathologic factors such as tumor size, vascular invasion, histologic grade, and the Ki-67 index to explore the clinical significances of E-cadherin and Prrx-1.

\section{MATERIALS AND METHODS}

\section{Patients and specimens}

A total of 244 patients who underwent surgical resection for HCC from 1991 to 2013 at Hanyang University Medical Center, Seoul and Guri hospitals were selected as subjects for this study. Medical records were reviewed to identify baseline clinical information, clinical course, underlying etiology, and liver function status at the diagnosis. Histological review was performed to determine stage, grade (Edmondson and Steiner's grading system), vascular invasion, size of tumor, and multiplicity. This study was conducted under review of the Institutional Review Board (IRB) of Hanyang University Guri Hospital (Code, 2015-11-012).

\section{TMA, immunohistochemistry, and interpretation}

Representative tissue blocks were selected for TMA construction after histological review. For each case, a 2-mm single core was punched from the tumor but not from normal tissue. All immunostainings were performed using the automated system, Leica Bond III (Leica Biosystems, Nusslock, Germany) and Bond Polymer Refine Detection Kit (Leica Biosystems). E-cadherin antibody (mouse monoclonal, NCL-L-E-Cad, Novocastra, Newcastle upon Tyne, UK) was diluted at 1:25. Prrx-1 antibody (rabbit polyclonal, NBP2-13816, Novus Biologicals, Littleton, CO, USA) was diluted at 1:20. Ki-67 antibody (mouse monoclonal, NCL-L-Ki67-MM1, Novocastra) was diluted at
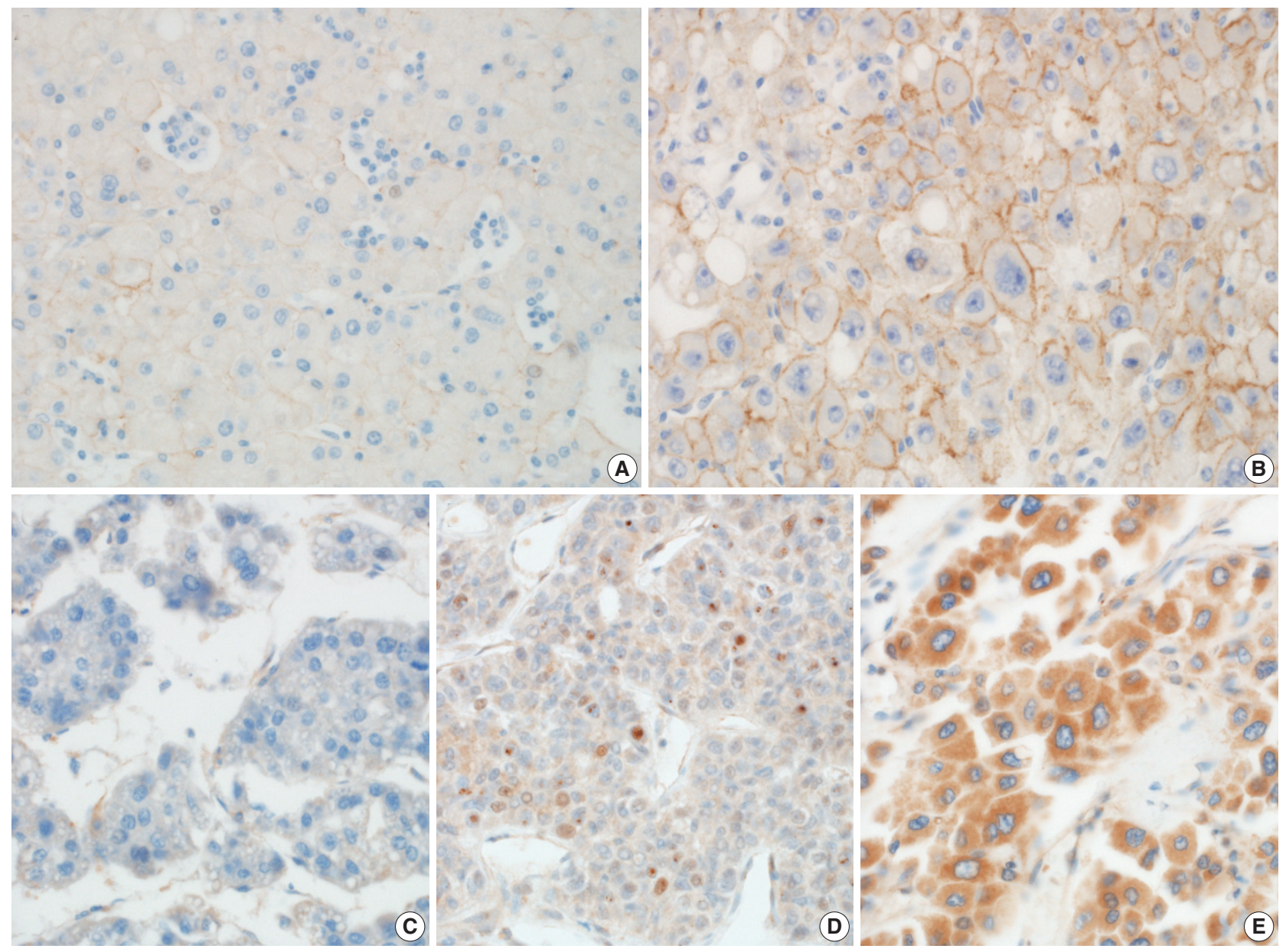

Fig. 1. Immunohistochemical staining of E-cadherin and paired-related homeobox protein 1 (Prrx-1) in hepatocellular carcinomas. Representative photos of immunohistochemical staining in hepatocellular carcinomas. (A) E-cadherin decreased. (B) E-cadherin maintained. (C) Prrx-1 negative. (D) Prrx-1 positive. (E) Prrx-1 negative, but cytoplasmic positive. 
1:200. TMA sections (4- $\mu$ m-thick) were de-waxed, and the antigen was retrieved with Bond Epitope Retrieval Solution 2 (Leica Biosystems), then incubated with primary antibodies for 60 minutes.

Two pathologists assessed E-cadherin staining into two categories as described previously; ${ }^{9}$ negative or faint membranous stained and moderate to strong membranous stained. The average E-cadherin stainability of HCC cells in each core was evaluated. Prrx-1 staining was evaluated by its nuclear positivity as previously described. ${ }^{4}$ Cytoplasmic stained cases without nuclear positivity for Prrx-1 were interpreted as negative because they were considered to be nonspecific cytoplasmic antibody entrapping. Representative images of immunohistochemical staining are presented in Fig. 1. To determine whether the immunoexpression of Prrx-1 in HCCs was upregulated or lost, comparing to normal or cirrhotic liver, we performed Prrx-1 immunostain to two whole block sections and one TMA section that contained 20 cores of normal liver tissue block obtained that were obtained from liver resections from traumatic liver laceration, or metastatic colon cancer. We stained one whole section from each of the three Prrx-1-positive HCCs and three Prrx-1-negative HCCs, to determine if the single TMA core was representative of the entire tumor's Prrx-1 expression status. The cases were randomly selected from the cohort and all sections included adjacent cirrhotic liver. Ki-67 immunostain was performed on TMA sections. The Ki-67 labeling index was calculated by a free image analyzing software, TMARKER v2.21625. ${ }^{10}$

\section{Statistical analysis}

We investigated the relationship between the loss of E-cadherin and clinicopathologic factors as well as survival time and recurrence. Expression of Prrx-1 was evaluated with the same method. Concordance of loss of E-cadherin and Prrx-1 expression was analyzed. We also correlated the expression with other markers to investigate whether those markers reflect aggressive pathologic features. The association between immunohistochemical stain results and clinicopathologic parameters was analyzed using the chi-square test, Fisher exact test, and Mann-Whitney $\mathrm{U}$ test. Recurrence and survival rate was calculated by the Kaplan-Meier method, and the comparisons were made using the log-rank test. The Cox proportional hazard regression model was adopted to determine the prognostic impact of immunohistochemical results and other clinicopathologic parameters. For the Cox regression, high $\mathrm{Ki}-67$ was defined as $\mathrm{Ki}-67$ index over 40 , by considering the cutoff values of previous studies. ${ }^{11,12}$ For all calculations, differences at $\mathrm{p}<.05$ were considered statis- tically significant. All statistical calculations were performed using the R software ver. 3.1.0 (R Core Team 2014).

\section{RESULTS}

\section{Clinicopathological characteristics}

The clinicopathologic features are summarized in Table 1. The median age of the 244 patients was 56 years (range, 24 to 87 years). One hundred eighty-three patients were male (75\%), and 61 were female $(25 \%)$. One hundred seventy-one patients had hepatitis $\mathrm{B}$ virus (HBV, 70.1\%), and 16 had hepatitis $\mathrm{C}$ virus (HCV, 6.6\%). Two patients had both HCV and HBV coinfection $(0.8 \%)$. Twenty-five patients had no viral hepatitis but had a history of chronic alcohol intake without any apparent vi-

Table 1. Clinicopathologic characteristics of HCC patients $(n=244)$

\begin{tabular}{|c|c|}
\hline Parameter & No. (\%) \\
\hline Age $(y r)^{a}$ & $56(24-87)$ \\
\hline Sex (male:female) & $183: 61(3: 1)$ \\
\hline \multicolumn{2}{|l|}{ Etiological factor } \\
\hline HBV & $171(70.1)$ \\
\hline $\mathrm{HCV}$ & $16(6.6)$ \\
\hline $\mathrm{HBV}+\mathrm{HCV}$ & $2(0.8)$ \\
\hline Alcohol & $25(10.2)$ \\
\hline Unknown & 30 (12.3) \\
\hline \multicolumn{2}{|l|}{ Underlying disease } \\
\hline Chronic hepatitis & $44(18)$ \\
\hline Cirrhosis & $200(82)$ \\
\hline \multicolumn{2}{|l|}{ Child-Pugh class } \\
\hline A & $230(94.3)$ \\
\hline B & $14(5.7)$ \\
\hline \multicolumn{2}{|l|}{ AJCC stage } \\
\hline I & $132(54.1)$ \\
\hline$\|$ & 79 (32.4) \\
\hline III & $33(13.5)$ \\
\hline Tumor size $(\mathrm{cm})^{a}$ & $3.5(0.6-19.5)$ \\
\hline \multicolumn{2}{|c|}{ Multiplicity (solitary/multiple) } \\
\hline Solitary & $201(82.4)$ \\
\hline Multiple & $43(17.6)$ \\
\hline \multicolumn{2}{|l|}{ Histologic grade } \\
\hline 1,2 & $109(44.7)$ \\
\hline 3,4 & $135(55.3)$ \\
\hline Small vessel invasion & $94(38.5)$ \\
\hline Large vessel invasion & $24(9.8)$ \\
\hline \multicolumn{2}{|l|}{ Recurrence rate (\%) } \\
\hline 1 Year & 35.0 \\
\hline 5 Years & 65.3 \\
\hline \multicolumn{2}{|l|}{ Survival rate (\%) } \\
\hline 1 Year & 87.4 \\
\hline 5 Years & 55.6 \\
\hline
\end{tabular}

HCC, hepatocellular carcinoma; HBV, hepatitis B virus; HCV, hepatitis C virus; AJCC, American Joint Committee on Cancer.

${ }^{\mathrm{a} M e d i a n}$ (range). 
ral etiology (10.2\%). Thirty patients had neither viral hepatitis nor history of chronic alcoholic intake (12.3\%). Cirrhosis was detected in 200 patients (82\%). Child-Pugh classes were stage $\mathrm{B}$ in 14 patients (5.7\%), while the others are stage $\mathrm{A}(94.3 \%)$. Regarding pathologic stage, 132 patients were stage I (54.1\%), 79 were II (33.4\%), eight were IIIA (3.3\%), 24 were IIIB (9.8\%), and one was IIIC (0.4\%). Median tumor size was $3.5 \mathrm{~cm}$ (range, 0.6 to $19.5 \mathrm{~cm}$ ). Two hundred one cases presented as a solitary tumor $(82.4 \%)$ while the others presented as multiple tumors. Portal vein, hepatic vein, or their major branch involvement was detected in 45 patients (18.4\%).
One hundred and six patients died during follow-up. Median survival time was 70.8 months (range, 1.3 to 287.7 months) and 5-year survival rate was $55.6 \%$. In addition, $35 \%$ of cases showed a tumor recurrence within 1 year, and $65.3 \%$ underwent recurrence within 5 years. The Kaplan-Meier method showed a significant difference in overall survival and diseasefree survival between stages I and II ( $\mathrm{p}=.006$, and $\mathrm{p}<.001$, respectively). However, those between stage II and stage III were not statistically significant $(\mathrm{p}=.450$ and $\mathrm{p}=.106$, respectively). Additionally, 3.9\% of stage I patients experienced tumor recurrence within 1 year $(n=19)$, compared with $20.6 \%$ of stage II ( $n$

Table 2. Association between E-cadherin and Prrx-1 expression and clinicopathologic parameters in HCC patients $(n=244)$

\begin{tabular}{|c|c|c|c|c|c|c|}
\hline \multirow{2}{*}{ Parameter } & \multicolumn{2}{|c|}{ E-cadherin } & \multirow{2}{*}{$p$-value } & \multicolumn{2}{|c|}{ Prrx-1 } & \multirow{2}{*}{$p$-value } \\
\hline & Decreased $(n=96)$ & Maintained $(n=148)$ & & Positive (n=32) & Negative $(n=212)$ & \\
\hline Age $(y r)^{a}$ & $57(31-77)$ & $55(24-87)$ & $.135^{\mathrm{b}}$ & $60(24-74)$ & $55.5(28-87)$ & $.161^{b}$ \\
\hline Sex (male:female) & $74: 22(3.4: 1)$ & $109: 39(2.8: 1)$ & $.650^{\circ}$ & $24: 8(3: 1)$ & $159: 53(3: 1)$ & $>.990^{\mathrm{C}}$ \\
\hline Etiology (\%) & & & $.090^{d}$ & & & \\
\hline HBV & $59(61.5)$ & $112(75.7)$ & & $20(62.5)$ & $151(71.2)$ & $.501^{d}$ \\
\hline $\mathrm{HCV}$ & $10(10.4)$ & $6(4.1)$ & & $2(6.2)$ & $14(6.6)$ & \\
\hline $\mathrm{HBV}+\mathrm{HCV}$ & $1(1)$ & $1(0.7)$ & & 0 & $2(0.8)$ & \\
\hline Alcohol & $13(13.5)$ & $12(8.1)$ & & $3(9.4)$ & $22(10.8)$ & \\
\hline Unknown & $13(13.5)$ & $17(11.5)$ & & $7(21.9)$ & $23(10.8)$ & \\
\hline Underlying disease (\%) & & & $.048^{\mathrm{C}}$ & & & $.893^{d}$ \\
\hline Chronic hepatitis & $11(11.5)$ & $33(22.3)$ & & $5(18.4)$ & $39(15.6)$ & \\
\hline Cirrhosis & 85 (88.5) & $115(77.7)$ & & 27 (81.6) & 173 (81.6) & \\
\hline Child-Pugh class & & & $.996^{\mathrm{c}}$ & & & $>.990^{d}$ \\
\hline A & $91(94.8)$ & $139(93.9)$ & & $30(93.8)$ & $200(94.3)$ & \\
\hline $\mathrm{B}$ & $5(5.2)$ & $9(6.1)$ & & $2(5.7)$ & $12(5.7)$ & \\
\hline AJCC stage & & & $.707^{\circ}$ & & & $.399^{d}$ \\
\hline 1 & $50(52.1)$ & $82(55.4)$ & & $20(62.5)$ & $112(52.8)$ & \\
\hline$\|$ & $34(35.4)$ & $45(30.4)$ & & $7(21.9)$ & $72(34.0)$ & \\
\hline III & $12(12.5)$ & $21(14.2)$ & & $5(15.6)$ & $28(13.2)$ & \\
\hline Multiplicity & & & $.406^{\mathrm{c}}$ & & & $.571^{\mathrm{c}}$ \\
\hline Single & $82(85.4)$ & $119(80.4)$ & & 28 (87.5) & $173(81.6)$ & \\
\hline Multiple & $14(14.6)$ & $29(19.6)$ & & $4(12.5)$ & $39(18.4)$ & \\
\hline Tumor size ${ }^{a}$ & $3.3(0.6-19.5)$ & $3.5(0.7-17)$ & $.877^{\mathrm{b}}$ & $3.15(1.2-15)$ & $3.5(0.6-19.5)$ & \\
\hline Histologic grade & & & $.224^{\circ}$ & & & $.286^{\circ}$ \\
\hline 1,2 & $48(50)$ & $61(41.2)$ & & $11(34.4)$ & 98 (46.2) & \\
\hline 3,4 & $48(50)$ & 87 (58.8) & & $21(65.6)$ & $114(53.8)$ & \\
\hline Large vessel invasion & $11(11.5)$ & $13(8.8)$ & $.642^{\mathrm{c}}$ & $4(12.5)$ & $20(9.4)$ & $.533^{\mathrm{d}}$ \\
\hline Small vessel invasion & $41(42.7)$ & $53(35.8)$ & $.344^{\mathrm{c}}$ & $13(40.6)$ & 81 (38.2) & $.946^{\mathrm{c}}$ \\
\hline Prrx-1 positivity & $12(12.5)$ & $20(13.5)$ & $.972^{\mathrm{C}}$ & $12(37.5)$ & $84(39.6)$ & $.972^{\mathrm{c}}$ \\
\hline Ki-67 index ${ }^{\mathrm{e}}$ & $21(9-37.5)$ & $22(10-41)$ & $.754^{\mathrm{b}}$ & $21(9-35.5)$ & $22(9.5-40.5)$ & $.533^{b}$ \\
\hline Recurrence rate (\%) & & & $<.001^{\dagger}$ & & & $.947^{\dagger}$ \\
\hline 1 Year & 45.8 & 28.5 & & 43.1 & 34.2 & \\
\hline 5 Years & 78.1 & 57.1 & & 71.2 & 64.8 & \\
\hline Survival rate (\%) & & & $.459^{f}$ & & & $.409^{\dagger}$ \\
\hline 1 Year & 88.3 & 86.8 & & 77.5 & 88.9 & \\
\hline 5 Years & 55.2 & 55.6 & & 66.3 & 55.1 & \\
\hline
\end{tabular}

Values are presented as number (\%) unless otherwise indicated.

Prrx-1, paired-related homeobox protein 1; HCC, hepatocellular carcinoma; HBV, hepatitis B virus; HCV, hepatitis C virus; AJCC, American Joint Committee on Cancer.

${ }^{\mathrm{a}}$ Median (range); ${ }^{\mathrm{b}}$ Mann-Whitney U test; ${ }^{\mathrm{c}}$ Chi-square test; ${ }^{\mathrm{d}}$ Fisher exact test; ${ }^{\mathrm{e}}$ Median (first quantile-third quantile); 'Log-rank test. 
$=19)$, and $29.8 \%$ of stage III ( $\mathrm{n}=11)$.

\section{E-cadherin and Prrx-1 immunohistochemical staining results}

E-cadherin expression was decreased in 96 cases (39.3\%), whereas others showed moderate to strong membranous immunoreactivity. Thirty-two cases (13.3\%) showed at least focal nuclear Prrx-1 immunoreactivity. Loss of E-cadherin and Prrx-1 expression was not concordant ( $\mathrm{p}=.972$ on the chi-square test). One hundred twenty cases showed minimally weak cytoplasmic stainability (49.2\%). Cytoplasmic staining of Prrx-1 was not correlated with loss of E-cadherin expression ( $\mathrm{p}=.217$ on the chisquare test).

All normal livers, including two whole section and one TMA section, of which there were twenty cases, were negative for Prrx1 staining. All Prrx-1 stainings with whole sections of three Prrx-1-positive HCCs and three Prrx-1-negative HCCs showed consistent results with the TMA stainings. All adjacent cirrhotic livers from the six sections were negative for Prrx- 1 .

\section{HCC biomarker relationships}

We examined the correlation between Prrx-1 and E-cadherin immunohistochemical staining results and various clinicopath- ological factors. HCCs with decreased E-cadherin tend to occur more frequently in the background of cirrhosis, compared to Ecadherin maintained HCCs $(88.5 \%$ vs $77.7 \%, p=.048$ on the chi-square test). Both large vessel (portal vein, hepatic vein, or their major branches) invasion and small vessel invasion were more frequent in the E-cadherin reduced group compared with the maintained group; however, they were not statistically significant (large vessel invasion: $11.5 \%$ vs $8.8 \%, \mathrm{p}=.642$ on chisquare test; small vessel invasion: $42.7 \%$ vs $35.8 \%, \mathrm{p}=.344$ on chi-square test). Hepatitis $B$, hepatitis $C$, history of chronic alcohol intake, age, sex, pathologic stage, multiplicity of the tumor, tumor size, and histologic grade were not significantly associated with E-cadherin expression (Table 2).

None of the factors were statistically significantly correlated with Prrx-1 expression and clinicopathological factors, although both large vessel invasion and small vessel invasion were more frequent in Prrx-1-positive HCCs (large vessel invasion: 12.5\% vs $9.4 \%, \mathrm{p}=.533$; small vessel invasion: $40.6 \%$ vs $38.2 \%$, $\mathrm{p}=$ .946) (Table 2). Prrx-1-positive HCCs showed more frequent $\mathrm{p} 53$ overexpression ( $25 \%$ vs $13.7 \%, \mathrm{p}=.114$ on chi-square test), although this result was not statistically significant.

Survival analysis indicated that E-cadherin decreased HCCs that were identified in earlier recurrences than in E-cadherin

Table 3. Association between E-cadherin and Prrx-1 expression as a combinational marker and clinicopathologic parameters in HCC patients $(n=244)$

\begin{tabular}{|c|c|c|c|c|}
\hline \multirow{2}{*}{ Parameter } & \multicolumn{2}{|c|}{ E-cadherin positive } & \multicolumn{2}{|c|}{ E-cadherin negative } \\
\hline & Prrx-1 positive $(n=20)$ & Prrx-1 negative $(n=128)$ & Prrx-1 positive $(n=12)$ & Prrx-1 negative $(n=84)$ \\
\hline \multicolumn{5}{|l|}{ AJCC stage } \\
\hline । & $11(55)$ & $73(57)$ & $9(75)$ & $41(48.8)$ \\
\hline$\|$ & $6(30)$ & 40 (31.2) & $1(8.3)$ & 33 (39.3) \\
\hline III & $3(15)$ & $15(11.7)$ & $2(16.7)$ & $10(11.9)$ \\
\hline \multicolumn{5}{|l|}{ Multiplicity } \\
\hline Single & $18(90)$ & $101(78.9)$ & $10(83.3)$ & $72(85.7)$ \\
\hline Multiple & $2(10)$ & 27 (21.1) & $2(16.7)$ & $12(14.3)$ \\
\hline \multicolumn{5}{|l|}{ Histologic grade ${ }^{a}$} \\
\hline 1,2 & $4(20)$ & $57(44.5)$ & $7(58.3)$ & $41(48.8)$ \\
\hline 3,4 & $16(80)$ & $71(55.5)$ & $5(41.7)$ & $43(51.2)$ \\
\hline Large vessel invasion & $18(90)$ & $117(91.4)$ & $10(83.3)$ & 75 (89.3) \\
\hline Small vessel invasion & $10(50)$ & $85(66.4)$ & $9(75)$ & $46(54.8)$ \\
\hline Ki-67 index ${ }^{b}$ & $26(7.5-43.5)$ & $21(10-40.5)$ & $21(12.5-39)$ & $21(9-34.5)$ \\
\hline \multicolumn{5}{|l|}{ Recurrence rate (\%) } \\
\hline 1 Year & 60.1 & 73 & 51.1 & 54.6 \\
\hline 5 Years & 54.1 & 43 & 15.3 & 23.2 \\
\hline \multicolumn{5}{|l|}{ Survival rate (\%) } \\
\hline 1 Year & 73.9 & 88.8 & 83.3 & 89 \\
\hline 5 Years & 54.6 & 56.9 & 83.3 & 51.7 \\
\hline
\end{tabular}

Values are presented as number (\%) unless otherwise indicated.

Prrx-1, paired-related homeobox protein 1; HCC, hepatocellular carcinoma; AJCC, American Joint Committee on Cancer.

${ }^{a}$ Comparing both E-cadherin and Prxx-1 positive-group (the first column) to the others, the former showed significantly higher histologic grade ( $p=.037$ on chisquared test); ${ }^{b}$ Median (first quantile-third quantile). 
Difference according to E-cadherin expression
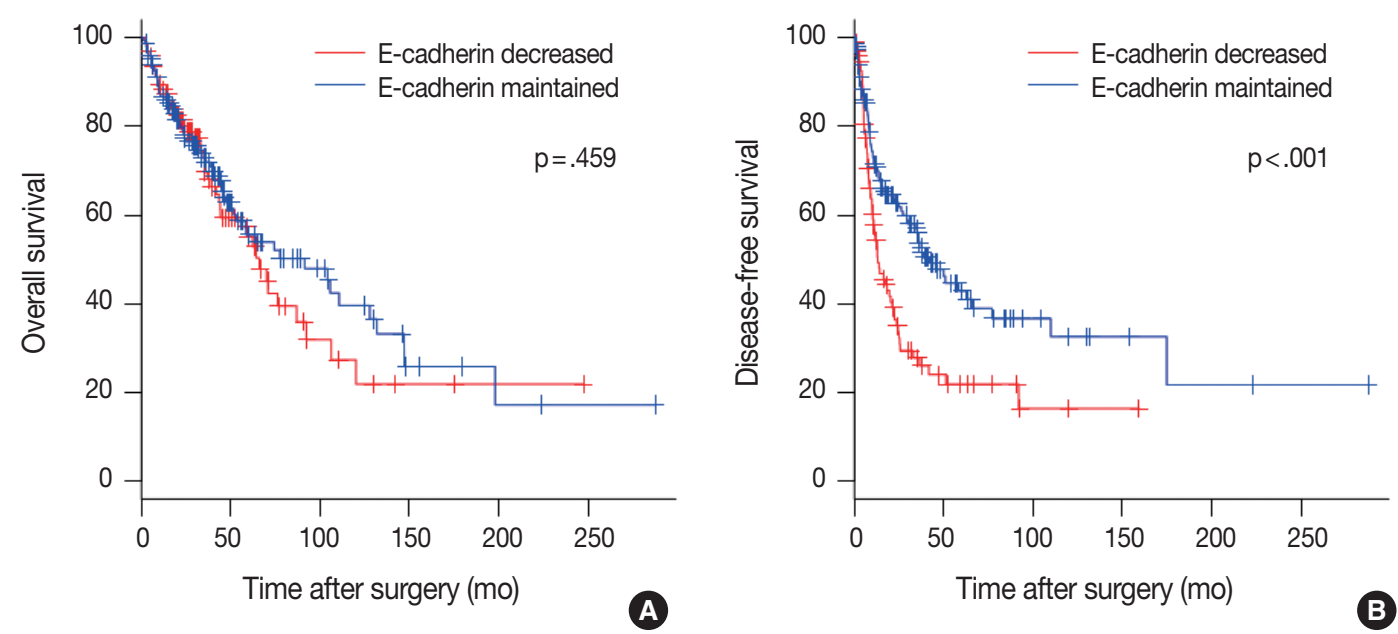

Difference according to Prrx-1 expression
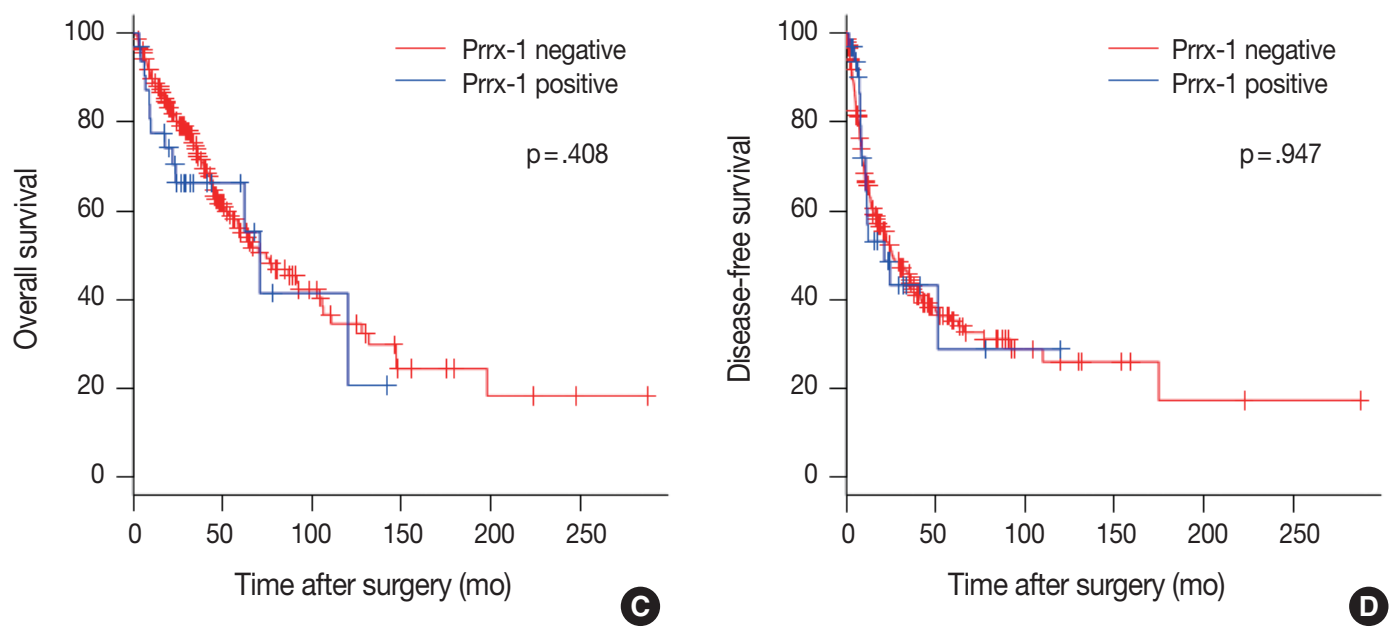

Four groups by combination of Prrx-1 and E-cadherin
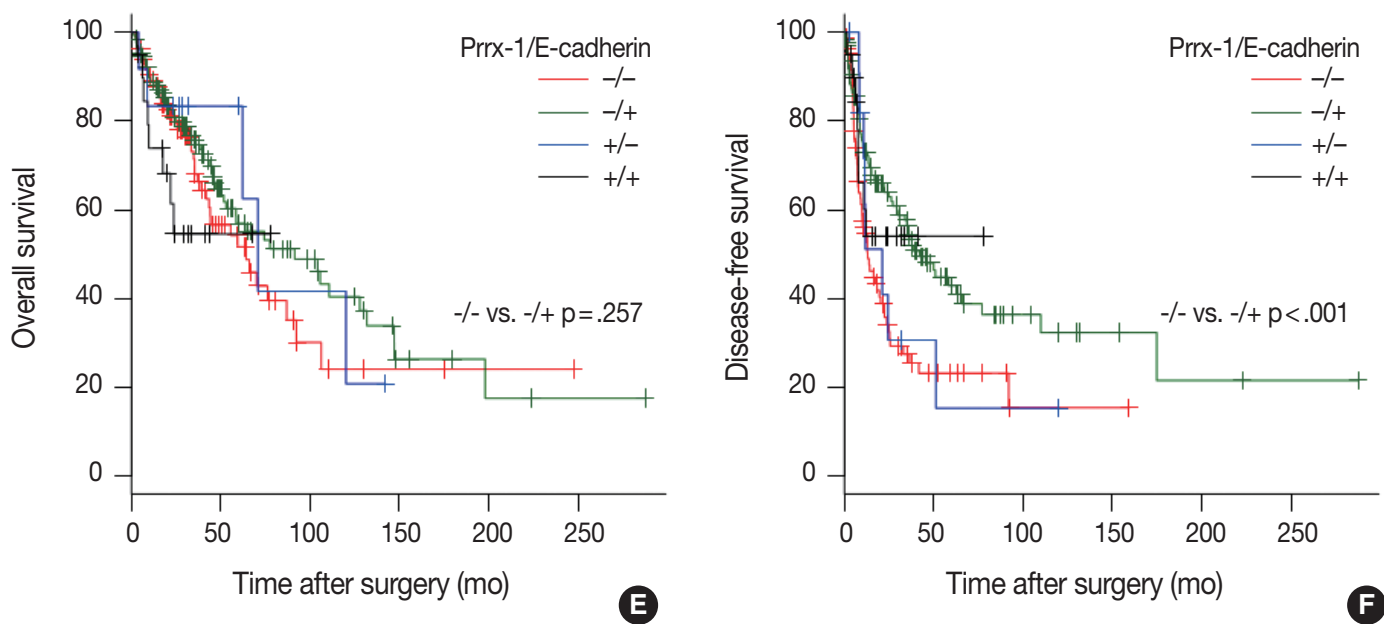

Fig. 2. Kaplan-Meier curves for overall survival $(A, C, E)$ and disease-free survival $(B, D, F)$ in hepatocellular carcinomas according to American Joint Committee on Cancer stage, E-cadherin and paired-related homeobox protein 1 (Prrx-1) expression (log-rank test). 
maintained HCCs (mean, 1.08 years vs 3.51 years; $\mathrm{p}<.001$ on the log-rank test) (Fig. 2B). Within one year, 41 cases (45.8\%) of E-cadherin decreased HCCs experienced recurrence, while 40 cases of E-cadherin (28.5\%) maintained HCCs did not. The overall survival rate was not significantly different on the logrank test $(\mathrm{p}=$.459) (Fig. 2A). We also examined the correlation between patient's survival/recurrence and Prrx-1 expression. Although 1-year recurrence rate ( $43.1 \%$ vs $34.2 \%$ ) and 5 -year recurrence rate (71.2\% vs $64.8 \%$ ) were higher in Prrx-1 that expressed HCCs than in Prrx-1-negative HCCs, the difference was not statistically significant on the log-rank test $(\mathrm{p}=.947)$ (Fig. 2D). Overall survival was also not significantly different $(\mathrm{p}=$ .408) (Fig. 2C).

We categorized HCCs into four groups, depending on the E- cadherin and Prrx-1 expression. The clinicopathologic parameters, recurrence rate, and survival rate were compared between Prrx-1-positive and Prrx-1-negative groups, and among E-cadherin-positive cases, in addition to the same comparison among E-cadherin-negative cases. Each of the four groups were also compared with the other three groups. The E-cadherin-positive and Prrx-1-positive group $(\mathrm{n}=20)$ showed significantly higher nuclear grades than the rest of the group $(n=224)$ (nuclear grade 3 or 4 proportion: $80 \%$ vs $53.1 \%, p=.037$ on chi-squared test). Other parameters in the various group comparison were not significantly different (Table 3, Fig. 2E and F).

\section{Cox proportional hazard regression model}

Factors that may affect the prognosis of HCC were filtered

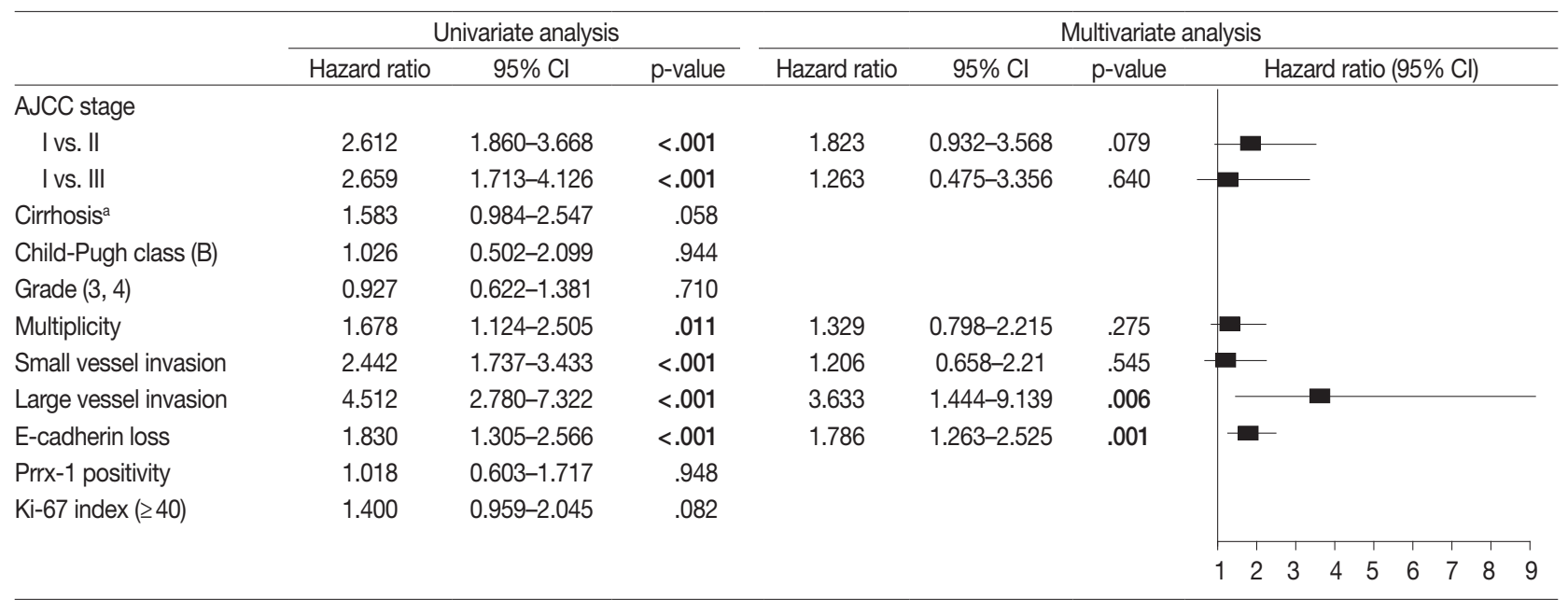

Fig. 3. Cox proportional hazard regression model for disease-free survival. Cl, confidence interval; AJCC, American Joint Committee on Cancer; Prrx-1, paired-related homeobox protein 1. ${ }^{a}$ Cirrhosis vs chronic hepatitis.

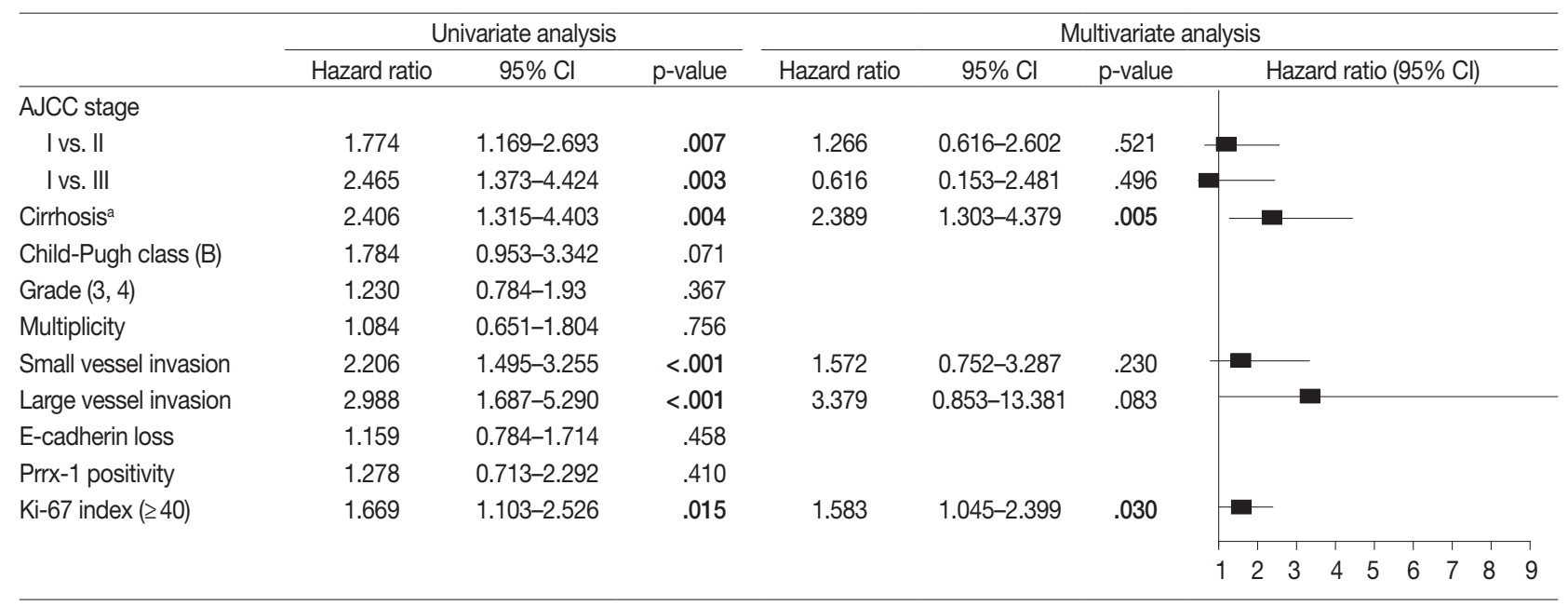

Fig. 4. Cox proportional hazard regression model for overall survival. Cl, confidence interval; AJCC, American Joint Committee on Cancer; Prrx-1, paired-related homeobox protein $1 .{ }^{2}$ Cirrhosis vs chronic hepatitis. 
for the multivariate Cox proportional hazard regression model $(\mathrm{p}<.05$ in univariate Cox proportional hazard regression model). The disease-free and overall survival were separately processed and are in Figs. 3 and 4, respectively. The stage, multiplicity, small and large vessel invasion, and decrease of E-cadherin were selected for factors in disease-free survival. Among them, the large vessel invasion $(\mathrm{p}=.006)$ and loss of E-cadherin $(\mathrm{p}=$ .001) were independent risk factors. The stage, cirrhosis, small and large vessel invasion, and high Ki-67 labeling index were selected for overall survival. Cirrhosis $(\mathrm{p}=.005)$ and high Ki-67 labeling index $(\mathrm{p}=.030)$ were independent risk factors for overall survival.

\section{DISCUSSION}

EMT has an important role in cancer progression because it influences tumor cell migration, allows local tissue invasion, and into the blood vessel, which is a necessary step in cancer metastasis. ${ }^{3}$ In colorectal cancer, tumor budding is one of the most important prognostic factors and reflects aggressive biologic behavior. The tumor budding is also explained as an EMT process, consistently with its morphologic change to fibroblast-like appearance. ${ }^{13}$ E-cadherin is a type of cadherins, which is a type 1 transmembrane protein, that plays an important role in cell adhesion. Loss of the E-cadherin is a hallmark of EMT and it is related to poor prognosis in various human cancers. ${ }^{14}$ In addition, several studies based on immunohistochemical staining of Ecadherin have reported the prognostic significance of HCC. ${ }^{15-17}$

Loss of E-cadherin is clearly associated with hematogenous tumor spread, lymph node metastasis, and local recurrence in various types of cancer, including breast cancer, colorectal cancer, and liver cancer..$^{18-20}$ For HCC, Chen et al. ${ }^{18}$ performed a meta-analysis to access the prognostic value of E-cadherin in HCC, and revealed that loss of E-cadherin was strongly associated with poor overall survival, recurrence-free survival, metastasis, vascular invasion, higher grade, and advanced TNM stage. ${ }^{18}$ As mentioned earlier, several other EMT inducers were evaluated as prognostic markers. SNAI1, an EMT inducer, was expressed in an invasive portion of HCC and was associated with the higher grade. Twist-1, another EMT inducer, was found to be associated with distant metastasis in HCC. ${ }^{21}$

As previously mentioned, either upregulation or downregulation of Prrx-1 has been reported to be associated with poor prognosis, depending on the type of cancer. In colorectal cancer, overexpression of PRRX1 mRNA was involved in metastasis and poor prognosis. ${ }^{7}$ In thyroid cancer, $57.7 \%$ of anaplastic thy- roid carcinomas and two of five hobnail variants of papillary carcinoma, which are very aggressive and associated with high mortality rate, were positive for Prrx-1 on immunohistochemical staining, while all conventional papillary carcinomas were negative. ${ }^{4}$ In comparison, Ocana et al. ${ }^{5}$ found that loss of Prrx-1 contributes to metastatic colonization and poor prognosis in breast cancer. They performed an experiment with a xenograft model, using human breast cancer cells that expressed both Prrx1 and Twist 1 . A knockdown of both Prrx-1 and Twist1 increased lung metastasis but not in a setting of only one knockdown. This indicated that downregulation of Prrx-1 is necessary to develop metastatic nodules and gain proliferative activity. ${ }^{5}$ Similarly, Hirata $e t$ al. ${ }^{8}$ studied mRNA expression level of Prrx-1 in HCC and found that the Prrx-1 downregulated group showed a poorer prognosis than the upregulated group. However, this study showed that normal livers did not express Prrx-1 protein immunohistochemically, and evokes a question that the decreased level of the PRRX1 mRNA has biological implications, as only a few (in our case, 13\%) of the HCCs showed nuclear immunoreactivity on Prrx-1.

$\mathrm{Ki}-67$ is a marker that reflects proliferative activity, which increases during carcinogenesis. Poorly differentiated HCCs show a higher index of Ki-67 than in well- to moderately-differentiated HCCs. ${ }^{22}$ In general, the proliferating activity decreases when tumor cells undergo EMT. ${ }^{23}$ We expected that loss of E-cadherin and expression of Prrx-1 would inversely correlate with the Ki-67 index. However, there was no significant difference, which was likely due to the limitation of a small number of Prrx1-positive cases and many other biological confounding variables that effect on the proliferation.

Through this study, we attempted to elucidate whether Prrx1-positive or Prrx-1-negative HCCs are associated with poor prognosis. As EMT is a dynamic process and its timely regulation is necessary to metastasis, it is necessary to develop new markers to know whether the tumor will re-gain proliferative and epithelial property after hematogenous spread, when we observe E-cadherin loss in the resected primary tumor. However, we only validated E-cadherin as a useful prognostic factor to predict recurrence, and Prrx-1 was not correlated with patient's prognosis and recurrence. Our experiment had some limitations, which were attributable, in part to the role of Prrx-1 on the prognosis of HCCs. The design of the present experiment did not implicate the complex mechanism of EMT-reverse EMT process during cancer metastasis. Cross-sectional studies using immunohistochemical staining on paraffin block have a limitation to dealing dynamic processes. Especially, as Prrx-1 is a transcription 
factor, two assumptions are required for this immunohistochemical study: (1) the expression of Prrx-1 should be consistent through the time until E-cadherin molecules disappear in the cell membrane, and (2) the functional expression range and threshold should be consistent with detection range and interpretation cutoff. However, many cellular signals work in a stochastic manner and show oscillatory responses, which give an uncertainty to our assumption. ${ }^{24,25}$ Prrx-1 overexpression was observed in $15.1 \%$ of HCCs in this study by immunohistochemistry. It is quite a small percentage comparing with $45.2 \%$ (28/62), which is the fraction of high-PRRX1 mRNA that expressed HCCs in the experiment by Hirata et $a l^{8}$ We believe that this also gives an uncertainty to our second assumption.

In summary, Prrx-1 was expressed in small portion (13.3\%) of HCCs but not in non-neoplastic livers. We validated that Ecadherin loss had the prognostic value to predict recurrence in HCCs. Prrx-1 was not correlated with EMT nor prognosis nor recurrence by immunohistochemical staining study. Further study with a larger series of HCCs is required to elucidate the significance of Prrx-1 in HCCs.

\section{Conflicts of Interest}

No potential conflict of interest relevant to this article was reported.

\section{REFERENCES}

1. Jung KW, Won YJ, Kong HJ, Oh CM, Seo HG, Lee JS. Cancer statistics in Korea: incidence, mortality, survival and prevalence in 2010. Cancer Res Treat 2013; 45: 1-14.

2. Tung-Ping Poon R, Fan ST, Wong J. Risk factors, prevention, and management of postoperative recurrence after resection of hepatocellular carcinoma. Ann Surg 2000; 232: 10-24.

3. Thiery JP, Acloque H, Huang RY, Nieto MA. Epithelial-mesenchymal transitions in development and disease. Cell 2009; 139: 871-90.

4. Hardin H, Guo Z, Shan W, et al. The roles of the epithelial-mesenchymal transition marker PRRX1 and miR-146b-5p in papillary thyroid carcinoma progression. Am J Pathol 2014; 184: 2342-54.

5. Ocana OH, Corcoles R, Fabra A, et al. Metastatic colonization requires the repression of the epithelial-mesenchymal transition inducer Prrx1. Cancer Cell 2012; 22: 709-24.

6. Reichert M, Takano S, von Burstin J, et al. The Prrx1 homeodomain transcription factor plays a central role in pancreatic regeneration and carcinogenesis. Genes Dev 2013; 27: 288-300.

7. Takahashi Y, Sawada G, Kurashige J, et al. Paired related homoeo- box 1, a new EMT inducer, is involved in metastasis and poor prognosis in colorectal cancer. Br J Cancer 2013; 109: 307-11.

8. Hirata H, Sugimachi K, Takahashi Y, et al. Downregulation of PRRX1 confers cancer stem cell-like properties and predicts poor prognosis in hepatocellular carcinoma. Ann Surg Oncol 2015; 22 Suppl 3: S1402-9.

9. Woo HY, Min AL, Choi JY, Bae SH, Yoon SK, Jung CK. Clinicopathologic significance of the expression of Snail in hepatocellular carcinoma. Korean J Hepatol 2011; 17: 12-8.

10. Schuffler PJ, Fuchs TJ, Ong CS, Wild PJ, Rupp NJ, Buhmann JM. TMARKER: A free software toolkit for histopathological cell counting and staining estimation. J Pathol Inform 2013; 4(Suppl): S2.

11. Nakanishi K, Sakamoto M, Yamasaki S, Todo S, Hirohashi S. Akt phosphorylation is a risk factor for early disease recurrence and poor prognosis in hepatocellular carcinoma. Cancer 2005; 103: 30712.

12. Ito $Y$, Matsuura N, Sakon M, et al. Both cell proliferation and apoptosis significantly predict shortened disease-free survival in hepatocellular carcinoma. Br J Cancer 1999; 81: 747-51.

13. Prall F. Tumour budding in colorectal carcinoma. Histopathology 2007; 50: 151-62.

14. Peinado H, Olmeda D, Cano A. Snail, Zeb and bHLH factors in tumour progression: an alliance against the epithelial phenotype? Nat Rev Cancer 2007; 7: 415-28.

15. Cho SB, Lee KH, Lee JH, et al. Expression of E- and N-cadherin and clinicopathology in hepatocellular carcinoma. Pathol Int 2008; 58: 635-42.

16. Zhang L, Huang G, Li X, et al. Hypoxia induces epithelial-mesenchymal transition via activation of SNAI1 by hypoxia-inducible factor -1alpha in hepatocellular carcinoma. BMC Cancer 2013; 13: 108.

17. Kwon GY, Yoo BC, Koh KC, Cho JW, Park WS, Park CK. Promoter methylation of E-cadherin in hepatocellular carcinomas and dysplastic nodules. J Korean Med Sci 2005; 20: 242-7.

18. Chen J, Zhao J, Ma R, Lin H, Liang X, Cai X. Prognostic significance of E-cadherin expression in hepatocellular carcinoma: a meta-analysis. PLoS One 2014; 9: e103952.

19. Aoki S, Shimamura T, Shibata T, et al. Prognostic significance of dysadherin expression in advanced colorectal carcinoma. Br J Cancer 2003; 88: 726-32.

20. Asgeirsson KS, Jonasson JG, Tryggvadóttir L, et al. Altered expression of E-cadherin in breast cancer. patterns, mechanisms and clinical significance. Eur J Cancer 2000; 36: 1098-106.

21. Zhao XL, Sun T, Che N, et al. Promotion of hepatocellular carcinoma metastasis through matrix metalloproteinase activation by epithelial-mesenchymal transition regulator Twist1. J Cell Mol Med 
2011; 15: 691-700.

22. Tannapfel A, Geissler F, Köckerling F, Katalinic A, Hauss J, Wittekind C. Apoptosis and proliferation in relation to histopathological variables and prognosis in hepatocellular carcinoma. J Pathol 1999; 187: 439-45.

23. Tsai JH, Donaher JL, Murphy DA, Chau S, Yang J. Spatiotemporal regulation of epithelial-mesenchymal transition is essential for squamous cell carcinoma metastasis. Cancer Cell 2012; 22: 725-36.

24. Ferrell JE Jr, Machleder EM. The biochemical basis of an all-or-none cell fate switch in Xenopus oocytes. Science 1998; 280: 895-8.

25. Batchelor E, Loewer A, Lahav G. The ups and downs of p53: understanding protein dynamics in single cells. Nat Rev Cancer 2009; 9:371-7. 